\title{
Penetrative convection due to absorption of radiation in a magnetic nanofluid saturated porous layer
}

https://doi.org/10.2478/sgem-2019-0018

received January 22, 2019; accepted May 8, 2019.

\begin{abstract}
The present study investigates the onset of penetrative convection in- duced by selective absorption of radiation in a magnetic nanofluid saturated porous medium. The influence of Brownian motion, thermophoresis, and magnetophoresis on magnetic nanofluid treatment is taken into consideration. The Darcy's model is selected for the porous medium. We conduct a linear stability analysis to examine the onset of instability and evaluate the results for two different configurations, namely, when the layer is heated from below and when the layer is heated from above. The numerical investigations are carried out by applying the Chebyshev pseudospectral method. The effect of the porosity parameter $E$, parameter $Y$ (represents the ratio of internal heating to boundary heating), Lewis number $L e$, concentration Rayleigh number $R n$, Langevin parameter $\alpha L$, width of nanofluid layer $d$, diffusivity ratio $\eta$, and modified diffusivity ratio $N A$ is examined at the onset of convection. The results indicate that the convection commences easily with an increase in the value of $Y, L e$, and $N A$ but opposite in the case with a decrease in the value of $E, \alpha L, \eta$ and $d$ for both the two configurations. The parameter $R n$ advances the onset of convection when the layer is heated from below, while delays the onset of convection when the layer is heated from above.
\end{abstract}

Keywords: Magnetic nanofluids; Penetrative convection; Darcy model; Radiation absorption; Magnetic field.

PACS Nos.: 44.40.+a; 47.20.-k; 47.56.+r; 47.65.Cb; 47.35.Tv; 47.65.-d

\footnotetext{
*Corresponding author: Mahesh Kumar Sharma, Department of Applied Sciences, National Institute of Technology Delhi, Delhi-110040, India, E-mail: mksharma.nit@gmail.com

Amit Mahajan: Department of Applied Sciences, National Institute of Technology Delhi, Delhi-110040, India
}

MSC(2000): 76E06; 76E25; 76S05; 76W05

\section{Introduction:}

Nanofluids are the liquids containing a suspension of nanoparticles (having a size typically in the range 1-100 $\mathrm{nm}$ ) in a base fluid. The base fluid can be an organic solvent or water, and the choice of base fluid depends on the applications for which the nanofluid is prepared. A wide range of applications for nanofluid can be found in several sectors including transportation, commercial, residential, industrial, and so on. Owing to the importance of nanofluids in these sectors, the area nanofluid convection in porous media attracted the attention of various researchers. A benchmark study of convective transport in nanofluid was organized by Buongiorno [1]. A new model was developed in his study, which consists of the effects of two important mechanisms, namely, Brownian diffusion and thermophoresis. There are several studies in which the phenomena related to the onset of nanofluid convection in porous media have been examined under different aspects. Using Buongiorno's model, Nield and Kuznetsov [2] analytically studied the onset of convection in a layer of porous medium saturated by a nanofluid. The authors observed that the value of the critical thermal Rayleigh number depends on the distribution (i.e., top-heavy or bottom-heavy) of nanoparticles. They reported that the oscillatory convection may occur in case of a bottom-heavy nanoparticle distribution. Later, this problem was re-examined by the same authors for a revised set of boundary conditions [3]. In their extended work, the authors considered the nanoparticle fraction in a way such that the nanoparticle flux is zero on the boundaries. The authors pointed out that the oscillatory convection can no longer occur with the choice of new boundary conditions. Using these more realistic boundary conditions, Yadav et al. [4] analyzed the thermal instability of rotating nanofluids. The authors mentioned that the 
model selected in their study is more realistic physically than those used in the previous studies (i.e., models with non-zero nanoparticle flux at boundaries). Different aspects of natural convection in porous media have been examined thoroughly by many researchers (see, e.g., Ref. [5-9]).

An experimental investigation of the onset of convection in a stably stratified fluid layer because of selective absorption of radiation was conducted by Krishnamurti [10]. The convection mechanism observed by Krishnamurti was a penetrative one, which was, stimulated by internal heating through absorption of radiation. Penetrative convection is a phenomenon that arises when buoyancy-driven motion penetrates into stratified layers [11]. In the experimental work, Krishnamurti considered a layer of water that contains a pH indicator known as thymol blue. This model of Krishnamurti was again investigated by Straughan [12] with the bounding surfaces being fixed as they will be in the experiment. The author reported that the results obtained in his study supports the work of Krishnamurti and the model developed by Krishnamurti is a very effective one. Hill [13] studied a modification of the system introduced by Krishnamurti [10] for a fluid-saturated porous medium. He used Darcy's model for the porous medium and performed both linear as well as nonlinear stability analysis. Later, the author again examined this system (i.e., Krishnamurti's [10] model) by considering Brinkman's model for the porous medium [14]. Chang [15] extended the study of Krishnamurti by considering a two-layer system in which a fluid layer overlays a porous layer. The author examined the problem for two different configurations, namely, when the layer is heated from below and when the layer is heated from above.

The effect of magnetic field on nanofluid convection has its relevance and significance in numerous applications in biochemical engineering, geophysics, astrophysics, and chemical engineering [16]. Yadav et al. [17] performed a linear stability analysis to investigate the effect of a uniform vertical magnetic field on the onset of nanofluid convection and discussed the case of both non-oscillatory as well as oscillatory convection. They reported that the parameters $\mathrm{Le}$ (nanofluid Lewis number), $N_{A}$ (modified diffusivity ratio), and $R n$ (concentration Rayleigh number) have a destabilizing effect on the system. Later, Gupta et al. [16] examined the onset of nanofluid convection subjected to an applied magnetic field. They considered a bottom-heavy nanoparticle distribution and discussed the stability analytically as well as numerically. The authors observed that the parameters $R n$ and $L e$ decelerate the onset of convection for both oscillatory and stationary mode of convection, whereas, the parameter $N_{A}$ advances the onset of convection for stationary convection and delays the onset of convection for oscillatory convection. For more interesting studies related to nanofluid convection subject to an applied magnetic field, the reader may refer to [18-22] and references therein. Magnetic nanofluids (MNFs or ferrofluids) are the nanofluids that consist of magnetic nanoparticles suspended in a non-magnetic base fluid. One of the most important characteristic features of the MNF that distinguishes MNF from other fluids is that the fluid flow in MNF can be controlled by selecting an appropriate magnetic field. In many of the applications, it is desirable to control the fluid flow in porous media without directly accessing the fluid. These applications include emplacement of geophysically imageable liquids into specific zones for subsequent imaging, treatment chemicals, or controlled positioning of liquids [23]. MNFs play a significant role in these applications. The study of ferrofluid convection in porous media subject to an applied magnetic field begins with the work of Vaidyanathan et al. [24]. Mahajan and Sharma [25] studied the convective instability in a MNF-layer-saturated porous medium subject to a uniform magnetic field. The impact of Brownian motion, thermophoresis, magnetophoresis, and Darcy's law on the MNF fluid flow is considered in their study. Sheikholeslami [26] investigated the free convection of MNF in a porous curved cavity subject to an external magnetic source. Several interesting problems related to the onset of magnetic nanofluid convection are investigated by [27-33] under different aspects.

The present literature survey confirms that there is no published work regarding the onset of MNF convection in porous media induced by selective absorption of radiation. In this article, a numerical investigation is performed to examine the onset of instability in a layer of MNF-saturated low-permeability porous media under the influence of an applied magnetic field. The results are discussed for various combinations of boundary conditions on impermeable surfaces, conducting surfaces, free surfaces, and surfaces with constant heat flux. In various engineering applications, the temperature of a wall is not uniform but, rather, is a result of the imposition of a constant heat flux [34]. These applications required the study of constant heat flux boundaries. Experimental studies with the constant heat flux boundaries are conducted by various researchers including $[35,36]$. In this article, we consider the following three different boundary conditions: (i) when both lower and upper boundaries are impermeable and conducting (I_C-I_C) 


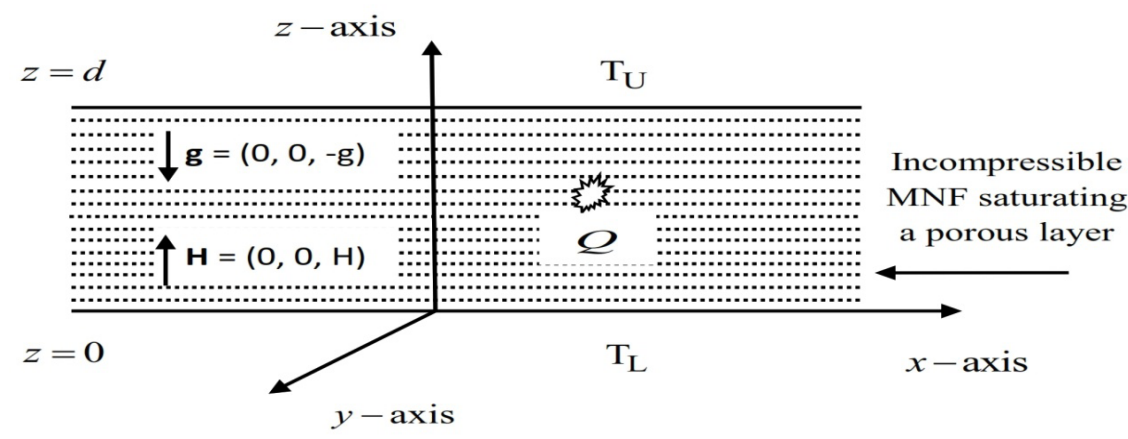

Figure 1: Physical configuration.

(ii) when lower boundary is impermeable and conducting while upper boundary is impermeable with constant heat flux (I_C-I_CHF), and (iii) when lower boundary is impermeable and conducting while upper boundary is free with constant heat flux (I_C-F_CHF). Moreover, the nanoparticle fraction is adjusted in a way such that the nanoparticle flux is zero on the boundaries. To derive the boundary conditions for nanoparticle volume fraction, the effect of both thermophoresis and magnetophoresis on the nanoparticle flux is taken into account. The results are calculated by using the Chebyshev pseudospectral method for two different configurations (i) when the layer is heated from below and (ii) when the layer is heated from above. The effects of several important parameters that affect the onset of instability are observed.

\section{Formulation of the problem}

The physical configuration of the problem is depicted in Figure 1. The model consists of a layer of incompressible, MNF-saturated, low-permeability, porous medium, subject to a uniform applied magnetic field $\mathbf{H}=H_{0}^{\text {ext }} \mathbf{k}$. The lower and upper boundaries are separated by thickness $d$ with temperature $T_{L}$ at the lower boundary and $T_{U}$ at the upper boundary. In addition, an internal heat source $Q$ is present within the MNF-saturated porous layer.

In order to derive the governing equations, the following assumptions are made [2]:

(1) fluid flow is incompressible,

(2) suspension is dilute $(\phi \square 1)$,

(3) viscous dissipation is negligible,

(4) two components (magnetic nanoparticles and base fluid) are locally in thermal equilibrium,

(5) the particles are suspended in nanofluid using either a surfactant or a surface charge technology that prevents particles from agglomeration and deposition on the porous matrix.

(6) the Boussinesq approximation and Darcy's law hold.

Following [3, 13, 37, 38], and under the above mentioned assumptions, the relevant equations governing the fluid flow are given as follows:

The equation of continuity is

$$
\nabla \cdot \mathbf{V}=0
$$

where $\mathbf{V}$ represents the filter velocity.

The equation of momentum is

$$
\frac{\rho_{f}}{E} \frac{\partial \mathbf{V}}{\partial t}=-\nabla p-\frac{\mu}{K} \mathbf{V}+\mu_{0}(\mathbf{M} . \nabla) \mathbf{H}-\rho g k,
$$

where $\rho_{f}, E, t, p, \mu, K, \mu_{0}$, and $\mathbf{M}$ are the density of MNF, porosity parameter, time, pressure term, viscosity, permeability parameter, magnetic permeability of vacuum and magnetization, respectively. Moreover, theMNFdensity $\rho$ is considered as $\rho=\phi \rho_{p}+(1-\phi) \rho_{f}\left(1-\alpha\left(T-T_{L}\right)\right)$. Here, we followed the model of Krishnamurti [10] and assumed that the density of fluid does not depend on the thymol blue concentration.

The equation of nanoparticle is

$$
\frac{\partial \phi}{\partial t}+\frac{1}{E} \mathbf{V} \cdot \nabla \phi=\nabla \cdot\left(D_{B} \nabla \phi+D_{T} \frac{\nabla T}{T_{L}}-D_{H} \frac{\nabla H}{H_{0}}\right)
$$

where $\phi$ is the volume fraction of magnetic nanoparticle. $D_{B}, D_{T}$, and $D_{H}$ stand for the Brownian diffusion, thermophoretic diffusion, and magnetophoretic coefficient, respectively. The term $H_{0}$ represents the uniform magnetic field of the MNF layer.

The equation of temperature is 


$$
\begin{aligned}
(\rho c)_{m}\left(\frac{\partial T}{\partial t}\right)+(\rho c)_{f}(\mathbf{V} \cdot \nabla T) & =\nabla \cdot\left(k_{1} \nabla T\right)+E(\rho c)_{p}\left(D_{B} \nabla T \cdot \nabla \phi\right. \\
& \left.+D_{T} \frac{\nabla T \cdot \nabla T}{T_{L}}-D_{H} \frac{\nabla T \cdot \nabla H}{H_{0}}\right)+Q,
\end{aligned}
$$

where $(\rho c)_{m},(\rho c)_{f}$, and $(\rho c)_{p}$ are the effective volumetric heat capacity of the porous medium, volumetric heat capacity for the MNF, and volumetric heat capacity for the nanoparticles, respectively. The terms $k_{1}$ and $Q$ represent the MNF thermal conductivity and heat source (which depends on the amount of radiation absorbed) respectively. Following Krishnamurti [10], a relationship between $Q$ and $C$ is considered in the following form: $Q=(\rho c)_{f} \alpha^{t} C$, where $\alpha^{t}$ represents a proportionality constant.

The equation of thymol blue concentration is

$$
\frac{\partial C}{\partial t}+\mathbf{V} \cdot \nabla C=k_{c} \nabla^{2} C
$$

where $C$ and $k_{c}$ are the concentration and thymol blue diffusivity, respectively.

The relevant Maxwell equations in the magnetostatic limit are taken as

$$
\nabla \cdot \mathbf{B}=0, \nabla \times \mathbf{H}=0, \mathbf{B}=\mu_{0}(\mathbf{M}+\mathbf{H}),
$$

where $\mathbf{B}$ represents the magnetic induction.

Following Kaloni and Lou [39], magnetization is assumed to be characterized as follows:

$$
M_{e q}=\frac{\mathbf{H}}{H} M_{s} \phi L\left(\alpha_{L}\right)=\frac{\mathbf{H}}{H} M_{e q}(H, T, \phi),
$$

where $L\left(\alpha_{L}\right)=\operatorname{coth}\left(\alpha_{L}\right)-\frac{1}{\alpha_{L}}$. Here $\alpha_{L}=\frac{m H}{k_{T} T}$ is the Langevin parameter. The terms ${ }^{{ }_{L}} \mathrm{k}_{B}, \mathrm{M}_{s}$, and ${ }^{B} m$ stand for the Boltzmann constant, magnetic saturation, and magnetic moment of a single particle, respectively.

To determine the solution in the quiescent state, the magnetic equation is linearized in the following manner [40]:

$$
\mathbf{M}=\frac{\mathbf{H}}{H}\left[M_{0}+\chi\left(H-H_{0}\right)-K_{m}\left(T-T_{h}\right)+K_{p}\left(\phi-\phi_{0}\right)\right],
$$

where $K_{m}=\chi H_{0} / T_{0}$ and $K_{p}=\chi H_{0} / \phi_{0}$ are the magnetic coefficients. The term $M_{0}$ and $\chi$ are the constant mean value of magnetization and tangent magnetic susceptibility respectively.
The parameters $\chi$ and $\chi_{2}$ (chord magnetic susceptibility) can be estimated by using the Langevin parameter as

$$
\alpha_{L}=\frac{m H_{0}}{k_{B} T_{0}}=\left\{\begin{array}{l}
\square 1, \quad \chi=\frac{M_{s} m}{3 k_{B} T_{0}}, \chi_{2}=\chi \\
\square=\frac{M_{s} m}{k_{B} T_{0}} L^{\prime}\left(\alpha_{L}\right), \chi_{2}=\frac{M_{s}}{T_{0}} L\left(\alpha_{L}\right) \\
\square 1, \quad \chi=\frac{M_{s} k_{B} T_{0}}{m H_{0}^{2}}, \chi_{2}=\frac{M_{s}}{H_{0}}\left(1-\frac{1}{\alpha_{L}}\right)
\end{array}\right.
$$

The boundary conditions are taken as

$$
\left.\begin{array}{l}
w=0, T=T_{L}, D_{B} \frac{\partial \phi}{\partial z}+\frac{D_{T}}{T_{L}} \frac{\partial T}{\partial z}-\frac{D_{H}}{H_{0}} \frac{\partial H}{\partial z}=0, C=C_{L} \quad \text { at } \quad z=0, \\
w=0, T=T_{U}, D_{B} \frac{\partial \phi}{\partial z}+\frac{D_{T}}{T_{L}} \frac{\partial T}{\partial z}-\frac{D_{H}}{H_{0}} \frac{\partial H}{\partial z}=0, C=0 \quad \text { at } \quad z=d .
\end{array}\right\}
$$

In addition, to derive the magnetic boundary conditions, the normal component of the magnetic induction and the tangential component of the magnetic field are assumed to be continuous across the boundary.

The dimensionless quantities are introduced as follows:

$\left(x^{*}, y^{*}, z^{*}\right)=(x, y, z) / d,\left(u^{*}, v^{*}, w^{*}\right)=(u, v, w) d / \kappa$,

$t^{*}=\left(\kappa / d^{2}\right) t, p^{*}=(K / \mu \kappa) p, T^{*}=\left(\sqrt{\frac{\rho_{f} g \alpha K d}{\mu \kappa\left|T_{L}-T_{U}\right|}}\right) T$,

$\phi^{*}=\frac{\phi}{\phi_{0}}, \mathbf{H}^{*}=\mathbf{H} / H_{0}, \mathbf{M}^{*}=\mathbf{M} / M_{0}$.

Note that $J \mid T_{L}-T_{U} \neq\left(T_{L}-T_{U}\right)$, where $J=\operatorname{sign}\left(T_{L}-T_{U}\right)$ which takes the value -1 when the layer is heated from above and +1 when the layer is heated from below.

Using equation (10) in equations (1)-(8), we obtain the following set of equations

$$
\nabla \cdot \mathbf{V}=0
$$

$$
\begin{aligned}
\frac{1}{V_{a}}\left(\frac{\partial \mathbf{V}}{\partial t}\right)=-\nabla p-\mathbf{V} & +\grave{u}(\mathbf{M} \cdot \nabla) \mathbf{H}-(R n \phi-R T \\
& \left.+R a_{N} \phi T-\gamma_{1} \phi+\gamma_{2}\right) \mathbf{k},
\end{aligned}
$$

$$
\frac{\partial \phi}{\partial t}+\frac{1}{E} \mathbf{V} \cdot \nabla \phi=\frac{1}{L e} \nabla^{2} \phi+\frac{N_{A}}{L e} \nabla^{2} T-\frac{N_{A}^{t}}{L e} \nabla^{2} H,
$$

$$
\begin{aligned}
A \frac{\partial T}{\partial t}+\mathbf{V} \cdot \nabla T=\nabla^{2} T & +\frac{N_{B}}{L e}(\nabla T \cdot \nabla \phi)+\frac{N_{A} N_{B}}{L e}(\nabla T \cdot \nabla T) \\
& -\frac{N_{A}^{t} N_{B}}{L e}(\nabla T \cdot \nabla H)+Y R C,
\end{aligned}
$$




$$
\begin{gathered}
\frac{\partial C}{\partial t}+\mathbf{V} \cdot \nabla \phi=\eta \nabla^{2} C, \\
\mathbf{M}=\frac{\mathbf{H}}{H} \frac{(1+\chi)}{\chi_{2}}\left\{\frac{\chi}{1+\chi} H-\frac{M_{1}}{M_{2}} T+\frac{M_{1}^{t}}{M_{2}^{t}} \phi+\frac{\chi_{2}-\chi}{1+\chi}\right\}, \\
\chi_{2} \nabla \cdot \mathbf{M}+\nabla \cdot \mathbf{H}=0 .
\end{gathered}
$$

In equations (12)-(17)

$$
\begin{aligned}
& R=\sqrt{\frac{\rho_{f} g \alpha d K\left|T_{L}-T_{U}\right|}{\mu \kappa}}, R n=\frac{\left(\rho_{p}-\rho_{f}\right) \phi_{0} g d K}{\mu \kappa}, \eta=\frac{k_{c}}{\kappa}, \operatorname{Pr}=\frac{\mu}{\rho_{f} \kappa}, \\
& N_{A}=\frac{D_{T}}{D_{B} T_{L} \phi_{0}} \sqrt{\frac{\mu \kappa\left|T_{L}-T_{U}\right|}{\rho_{f} g \alpha d K}}, N_{A}^{t}=\frac{D_{H}}{D_{B} \phi_{0}}, Y=\frac{\alpha^{t} C_{L} d^{2}}{\kappa\left|T_{L}-T_{U}\right|} \\
& N_{B}=E \frac{(\rho c)_{p}}{(\rho c)_{f}} \phi_{0}, L e=\frac{\kappa}{D_{B}}, M_{1}=\frac{\mu_{0} \chi^{2} H_{0}^{2}}{\rho_{f} g \alpha d(1+\chi) T_{L}^{2}} \sqrt{\frac{\mu \kappa\left|T_{L}-T_{U}\right|}{\rho_{f} g \alpha d K}}, \\
& M_{1}^{t}=\frac{\mu_{0} \chi^{2} H_{0}^{2}}{\rho_{f} g \alpha d(1+\chi) \phi_{0}}, M_{2}=\frac{\mu_{0} \chi H_{0}^{2}}{\rho_{f} g \alpha d T_{h}}, M_{2}^{t}=\frac{\mu_{0} \chi H_{0}^{2}}{\rho_{f} g \alpha d \phi_{0}},
\end{aligned}
$$

are the square root of thermal Rayleigh number, concentration Rayleigh number, diffusivity ratio, Prandtl number, modified diffusivity ratios $\left(N_{A}, N_{A}^{t}\right)$, ratio of internal heating to boundary heating, modified particledensity increment, MNF Lewis $t$ number, and magnetic parameters $\left(M_{1}, M_{1}, M_{2}, M_{2}\right)$, respectively. In addition, the parameters $\gamma_{1}=\left(\rho_{f} g \phi_{0} \alpha d K T_{L}\right) /(\mu \kappa)$, $\gamma_{2}=\left(\rho_{f}\left(1+\alpha T_{L}\right) g d K\right) /(\mu \kappa), \quad R a_{N}=\phi_{0} R \quad$ and $\mathrm{\textrm {u }}=\left(\mu_{0} M_{0} H_{0} K\right) /(\mu \kappa)$ stand for some non-dimensional group. Note that, the thermal Rayleigh number for the present problem is defined as $R a=R^{2}$.

In dimensionless form, the boundary conditions (equation (9)) take the following form:

$$
\left.\begin{array}{l}
w=0, T=T_{L} \sqrt{\frac{\rho_{f} g \alpha d^{3}}{\mu \kappa \mid T_{L}-T_{U}}} \frac{\partial \phi}{\partial z}+N_{A} \frac{\partial T}{\partial z}-N_{A}^{t} \frac{\partial H}{\partial z}=0, C=1, \text { at } \quad z=0, \\
w=0, T=T_{U} \sqrt{\frac{\rho_{f} g \alpha d^{3}}{\mu \kappa \mid T_{L}-T_{U}}} \frac{\partial \phi}{\partial z}+N_{A} \frac{\partial T}{\partial z}-N_{A}^{t} \frac{\partial H}{\partial z}=0, C=0, \text { at } \quad z=1 .
\end{array}\right\}
$$

\section{The basic state}

The basic state solution is assumed to be in the following form:

$$
\begin{aligned}
& \mathbf{v}=\mathbf{0}, p=p_{b}(z), T=T_{b}(z), \phi=\phi_{b}(z), \\
& C=C_{b}(z), \mathbf{M}=\mathbf{M}_{b}(z), \mathbf{H}=\mathbf{H}_{b}(z) .
\end{aligned}
$$

Using (19), equations (12)-(17) become

$$
\begin{gathered}
-\frac{d p_{b}}{d z}+\mathrm{ù} M_{b} \frac{d H_{b}}{d z}-R n \phi_{b}+R T_{b}-R a_{N} T_{b} \phi_{b}+\gamma_{1} \phi_{b}-\gamma_{2}=0, \\
\frac{d^{2} \phi_{b}}{d z^{2}}+N_{A} \frac{d^{2} T_{b}}{d z^{2}}-N_{A}^{t} \frac{d^{2} H_{b}}{d z^{2}}=0, \\
\frac{d^{2} T_{b}}{d z^{2}}+\left\{\frac{N_{B}}{L e} \frac{d \phi_{b}}{d z}+\frac{N_{A} N_{B}}{L e} \frac{d T_{b}}{d z}-\frac{N_{A}^{t} N_{B}}{L e} \frac{d H_{b}}{d z}\right\} \frac{d T_{b}}{d z}+Y R C_{b}=0, \\
\frac{d^{2} C_{b}}{d z^{2}}=0, \\
\chi_{2} \frac{d M_{b}}{d z}+\frac{d H_{b}}{d z}=0, \\
M_{b}=\frac{1+\chi}{\chi_{2}}\left\{\frac{\chi}{1+\chi} H_{b}-\frac{M_{1}}{M_{2}} T_{b}+\frac{M_{1}^{t}}{M_{2}^{t}} \phi_{b}+\frac{\chi_{2}-\chi}{1+\chi}\right\} .
\end{gathered}
$$

Solving equations (20)-(25) subject to boundary conditions (equation (18)) gives

$$
\begin{aligned}
& T_{b}=G(z)+\frac{T_{L}}{\mid T_{L}-T_{U}} R, \phi_{b}=\phi_{0}-\frac{D_{1}}{D_{2}} G(z), C_{b}=1-z, \\
& H_{b}=1+\left(\frac{M_{1}}{M_{2}}+\frac{D_{1}}{D_{2}} \frac{M_{1}^{t}}{M_{2}^{t}}\right) G(z), M_{b}=1-\frac{1}{\chi_{2}}\left(\frac{M_{1}}{M_{2}}+\frac{D_{1}}{D_{2}} \frac{M_{1}^{t}}{M_{2}^{t}}\right) G(z),
\end{aligned}
$$

Figure 2 represents the base flow profiles of $T_{b}, \phi_{b}, H_{b}$, and $\mathrm{M}_{b}$. The figure is plotted for two different configurations, namely, when the layer is heated from below $(J=+1)$ and when the layer is heated from above $(J=-1)$. It can be seen from the figure that the base flow profiles of $T_{b}, \phi_{b}, H_{b}$, and $M_{b}$ for $J=+1$ are completely different from the base flow profiles plotted for $J=-1$.

\section{The linear stability problem}

Now, we have superimposed the infinitesimally small perturbation on the basic state solution in the following form:

$$
\begin{array}{r}
{[\mathbf{v}, p, \phi, T, C, \mathbf{H}, \mathbf{M}]=\left[\mathbf{v}^{t}, p_{b}+p^{t}, \phi_{b}+\phi^{t}, T_{b}+\theta^{t},\right.} \\
\\
\left.C_{b}+C^{t}, \mathbf{H}_{b}+\mathbf{H}^{t}, \mathbf{M}_{b}+\mathbf{M}^{t}\right],
\end{array}
$$

where $\mathbf{v}^{t}, p^{t}, \phi^{t}, \theta^{t}, C^{t}, \mathbf{H}^{t}$, and $\mathbf{M}^{t}$ are the perturb variables that are considered to be small. 

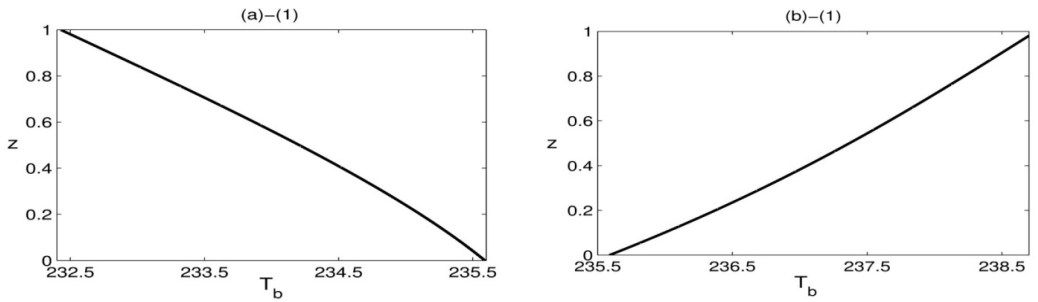

(a)-(2)
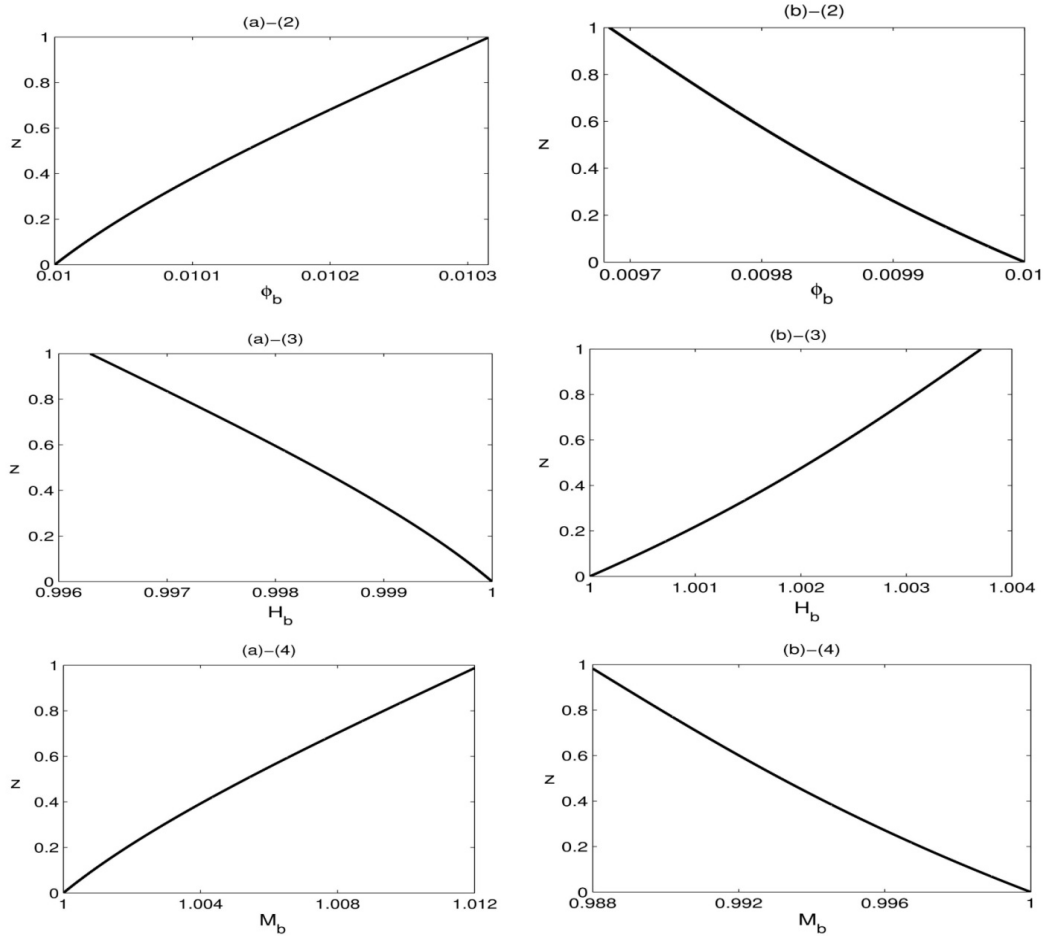

Figure 2: Base flow profile of (1) $T_{b}$, (2) $\phi_{b}$, (3) $\mathrm{H}_{b}$, and (4) $M_{b}$ for (a) $J=+1$, and (b) $J=-1$.

where $D_{1}=N_{A}-N_{A}^{\prime} \frac{M_{1}}{M_{2}}, \quad D_{2}=1+N_{A}^{\prime} \frac{M_{1}^{t}}{M_{2}^{t}}$,

and $G(z)=Y R\left(\frac{z^{3}}{6}-\frac{z^{2}}{2}\right)+R\left(\frac{Y}{3}-J\right) z$

Using equation (27) into equations (12)-(17) yields

$$
\begin{aligned}
A \frac{\partial \theta}{\partial t}= & -G^{\prime}(z) w+\nabla^{2} \theta-\left\{\frac{N_{B}}{L e} \frac{D_{1}}{D_{2}} G^{\prime}(z)-\frac{2 N_{A} N_{B}}{L e} G^{\prime}(z)\right. \\
& \left.+\frac{N_{B} N_{A}^{t} M_{1}}{L e M_{2}} G^{\prime}(z)+\frac{N_{B} N_{A}^{t} M_{1}^{t}}{L e M_{2}^{t}} \frac{D_{1}}{D_{2}} G^{\prime}(z)\right\} \frac{\partial \theta}{\partial z} \\
& +\frac{N_{B}}{L e} \frac{\partial \phi}{\partial z} G^{\prime}(z)-\frac{N_{B} N_{A}^{t}}{L e} G^{\prime}(z) \frac{\partial^{2} \psi}{\partial z^{2}}, \\
\frac{\partial C}{\partial t} & =w+\eta \nabla^{2} C,
\end{aligned}
$$$$
\times \nabla_{1}^{2} \theta-\left\{R a_{N} G(z)-R \frac{M_{2} M_{1}^{t}}{M_{2}^{t}} G^{\prime}(z)-R a_{s} M_{1}^{t} \frac{D_{1}}{D_{2}} G^{\prime}(z)+R n\right\} \nabla_{1}^{2} \phi
$$$$
+\left(M_{2} R+R a_{s} M_{2}^{t} \frac{D_{1}}{D_{2}}\right) G^{\prime}(z) \frac{\partial \nabla_{1}^{2} \psi}{\partial z},
$$

$$
\frac{\partial^{2} \psi}{\partial z^{2}}=\frac{M_{1}}{M_{2}} \frac{\partial \theta}{\partial z}-\frac{M_{1}^{t}}{M_{2}^{t}} \frac{\partial \phi}{\partial z}-\frac{1+\chi_{2}}{1+\chi} \nabla_{1}^{2} \psi
$$


Equations (28)-(32) possesses a boundary value problem Page. We reset the present domain from $[0,1]$ to $[-1,1]$ by applying a coordinate transformation from $z$ to $2 z-1$ in equations (28)-(32). This is so because, here we have planned to apply the Chebyshev pseudospectral method in which the domain of the problem must be $[-1,1]$. Thereafter, we use the normal mode procedure in which the normal mode solution is considered to be in the following form:

$$
\begin{aligned}
\{w, \theta, \phi, \psi, C\} & =\{w(z), \theta(z), \phi(z), \psi(z), C(z)\} \\
& \times \exp \left\{\sigma t+i\left(k_{x} x+k_{y} y\right)\right\},
\end{aligned}
$$

where $k_{x}$ and $k_{y}$ represent the wave numbers in $x$ -direction and $y$-direction, respectively.

Using equation (33), equations (28)-(32) give the following system of equations

$$
\begin{aligned}
& \frac{\sigma}{V_{a}\left(4 D^{2}-k^{2}\right) w(z)}=-\left(4 D^{2}-k^{2}\right) w(z)+\left\{M_{1} R G^{\prime}\left(z_{1}\right)-R+R a_{N} \phi_{0}\right. \\
&\left.-R a_{N} \frac{D_{1}}{D_{2}} G\left(z_{1}\right)+R \frac{M_{2} M_{1}^{t}}{M_{2}^{t}} \frac{D_{1}}{D_{2}} G^{\prime}\left(z_{1}\right)\right\} k^{2} \theta(z) \\
&+\left\{R a_{N} G\left(z_{1}\right)-R \frac{M_{2} M_{1}^{t}}{M_{2}^{t}} G^{\prime}\left(z_{1}\right)-R a_{s} M_{1}^{t} \frac{D_{1}}{D_{2}} G^{\prime}\left(z_{1}\right)\right. \\
&+R n\} k^{2} \phi(z)-\left(R M_{2}+R a_{s} M_{2}^{t} \frac{D_{1}}{D_{2}}\right) 2 G^{\prime}\left(z_{1}\right) k^{2} D \psi(z), \\
& \sigma \phi(z)=G^{\prime}\left(z_{1}\right) \frac{D_{1}}{D_{2}} w+\frac{1}{L e}\left(4 D^{2}-k^{2}\right) \phi+\frac{N_{A}}{L e}\left(4 D^{2}-k^{2}\right) \theta-2 \frac{N_{A}^{t}}{L e} D\left(4 D^{2}-k^{2}\right) \psi, \\
& \sigma \theta(z)=-G^{\prime}\left(z_{1}\right) w+\left(4 D^{2}-k^{2}\right) \theta(z)-2\left\{\frac{N_{B}}{L e} \frac{D_{1}}{D_{2}} G^{\prime}\left(z_{1}\right)\right. \\
&-\left.\frac{2 N_{A} N_{B}}{L e} G^{\prime}\left(z_{1}\right)+\frac{N_{B} N_{A}^{t} M_{1}}{L e M_{2}} G^{\prime}\left(z_{1}\right)+\frac{N_{B} N_{A}^{t} M_{1}^{t}}{L e M_{2}^{t}} \frac{D_{1}}{D_{2}} G^{\prime}\left(z_{1}\right)\right\} \\
& \times D \theta+2 \frac{N_{B}}{L e} G^{\prime}\left(z_{1}\right) D \phi-4 \frac{N_{B} N_{A}^{t}}{L e} G^{\prime}\left(z_{1}\right) D^{2} \psi, \\
& \sigma C(z)=w+\eta\left(4 D^{2}-k^{2}\right) C(z), \\
& 4 D^{2} \psi= 2 \frac{M_{1}}{M} D \theta-2 \frac{M_{1}^{t}}{M_{2}^{t}} D \phi+\left(\frac{1+\chi_{2}}{1+\chi}\right) k^{2} \psi .
\end{aligned}
$$

The boundary conditions for the amplitudes take the form

$$
\left.\begin{array}{c}
D \phi+N_{A} D \theta-2 N_{A}^{t} D^{2} \psi=C=0 \quad \text { at } \quad z= \pm 1, \\
2(1+\chi) D \psi-k \psi=0 \quad \text { at } \quad z=-1, \\
2(1+\chi) D \psi+k \psi=0 \quad \text { at } \quad z=+1,
\end{array}\right\}
$$

with $w=\theta=0$ at $z= \pm 1$ for I_C $-\mathrm{I} \_\mathrm{C}$ boundaries; $w=\theta=0$ at $z=-1$ and $w=\bar{D} \theta=\overline{0}$ at $z=+1$ for I_C - I_CHF boundaries; $w=\theta=0$ at $z=-1$ and $D \bar{w}=D \theta=0$ at $z=+1$ for I_C $-\mathrm{F}_{-} \mathrm{CHF}$ boundaries.

\section{Method of solution}

The above-mentioned system of equations (34)-(38) with the boundary conditions (equation (39)) constitutes an eigenvalue problem. The Chebyshev pseudospectral method is selected to solve this eigenvalue problem. We closely followed the same process and algorithm, as mentioned in Ref. [39]. First, we applied QZ-algorithm to calculate the salient eigenvalue (say $\sigma=\sigma_{r}+i \sigma_{j}$ ) for fixed values of $k, \alpha_{L}, L e, R n, Y, \eta$, and several other dimensionless parameters. By the salient eigenvalue, we mean that eigenvalue which has the largest real part. Second, we applied the Regula Falsi method to determine the particular value of $\beta$ corresponding to which the real part $\sigma_{r}$ of the salient eigenvalue $\sigma$ tends to zero. This procedure endows a single point in the neutral stability curve. We reiterate this procedure for several values of $k$ to enlist the desired neutral stability curve. The critical temperature gradient $\beta_{c}$ with $k_{c}$ (the critical wave number) can be defined as

$$
\beta_{c}=\min _{k} \beta\left(Y, \eta, \alpha_{L}, \operatorname{Pr}, L e \ldots\right)
$$

Moreover, the function FMINBND (a combination searching of gold section and parabolic method) of MATLAB is used to minimize equation (40).

To examine the nature of the stability, we applied a numerical approach by using the Chebyshev pseudospectral method. The nature of the stability is called stationary (or non-oscillatory) if the imaginary part of the salient eigenvalue tends to be zero at the same time when its real part approaches to zero. Otherwise, the nature of the stability is called oscillatory. In order to have a check on the nature of the stability, the salient eigenvalue $\sigma=\sigma_{r}+i \sigma_{j}$ is estimated one by one for all the dimensionless parameters and noticed that $\sigma_{j}$ always tends to be zero simultaneously when $\sigma_{r}$ tends to be zero. This behavior is found to be same for both the configurations (i.e., $J=+1$ and $J=-1$ ). Thus the nature of the stability for the present problem is stationary.

The present numerical solution is validated by comparing the results with those in Hill [13]. For this purpose, we have solved our problem in the absence of a 
Table 1: Comparison of $R a_{c}$ and $k^{2}$.

\begin{tabular}{llllll}
\hline & & Hill [13] & Present study & \\
\hline$J$ & $\eta$ & $k_{c}{ }^{2}$ & $R a_{c}$ & $k_{c}^{2}$ & $R a_{c}$ \\
\hline+1 & 0.001 & 4.9720 & 0.647667688 & 4.9720 & 0.647666602 \\
& 0.005 & 5.1174 & 3.054321113 & 5.1175 & 3.054321508 \\
& 0.01 & 5.2908 & 5.699734754 & 5.2900 & 5.699684893 \\
& 0.001 & 4.8990 & 0.667125786 & 4.8991 & 0.667125722 \\
& 0.005 & 4.7525 & 3.541442825 & 4.7524 & 3.541426234 \\
& 0.01 & 4.5617 & 7.666775544 & 4.5616 & 7.666778591 \\
\hline
\end{tabular}

magnetic field and magnetic nanoparticle concentration for I_C-I_C boundaries. It can be seen from Table 1 that the comparison is found to be very good.

\section{Results and discussion}

In this section, the results are illustrated graphically in Figures 3-7 and in Table 2 for $J=+1$ and $J=-1$. The results are derived for the following nondimensional parameters that significantly affect the onset of convection: porosity parameter, the ratio of internal heating to boundary heating, Lewis number, concentration Rayleigh number, Langevin parameter, the width of MNF layer, the diffusivity ratio, and modified diffusivity ratio. Following Kaloni and Lou [39] [Table 1, page 7] and Rosensweig [41] [Table 2.4, page 71], the values of pertaining parameters are taken as $\rho_{f}=1,180, k_{1}=0.59, M_{s}=15,900, \mu=0.007, \alpha=5.2 e-4$.

In order to illustrate the influence of selective absorption of radiation at the onset of MNF convection, the neutral stability curves for different values of $Y$ are plotted in Figure 3 for three different boundary conditions. For both the cases, namely, $J=+1$ and $J=-1$, it can be seen from the figure that as the value of $Y$ increases, the value of $R a_{c}$ decreases for all the three boundary conditions. It elucidates that the parameter $Y$ advances the onset of convection. The reason for such behavior of $Y$ is the following: a higher value of $Y$ implies that the internal heating is promoted, which in turn creates a distprbance in the MNF layer and leads to a lower value of ${ }^{c}$. This finding agrees well with the result of Hill [13] in the absence of a magnetic field and nanoparticle concentration.

To examine the influence of porous medium and Lewis number on the stability of the system, we plot the variation of $R a_{c}$ as a function of porosity parameter $E$ for several values of $L e$ in Figure 4. It is clear from the figure that $R a_{c}$ increases as $E$ increases. Thus, an increase in the parameter $E$ decelerates the onset of MNF convection. Such behavior of epsilon is in agreement with the literature by Yadav et al. [7, 42] in the absence of an applied magnetic field. It is also observed from the figure 4 that the values of $R a_{c}$ decreases as $L e$ increases. As the Lewis number is directly proportional to the thermal diffusivity, therefore, as the value of $L e$ increases, the value of thermal diffusivity also increase. For a higher value of thermal diffusivity, the amplitude of disturbance waves increases, which leads to a lower value of $R a_{c}$ [43]. Thus the parameter $L e$ advances the onset of MNF convection. A similar observation was made earlier by Yadav et al. [7] in the absence of an applied magnetic field.

In the primary study of nanofluid convection in porous media, Nield and Kuznetsov [2] reported that the stabilizing or destabilizing behavior of $R n$ depends on the type of nanoparticle distribution (i.e., whether nanoparticle distribution is bottom-heavy or top-heavy). Neutral stability curves for three different boundary conditions are presented in Figure 5 for several values of $R n$. The graphs are plotted for two different configurations, namely, $J=+1$ (i.e., when the layer is heated from below) and $J=-1$ (i.e., when the layer is heated from above). It can be seen from Figure 5 that the value of $R a_{c}$ decreases as $R n$ increases for $J=+1$, whereas the value of $R a_{c}$ increases as $R n$ increases for $J=-1$. The reason behind this may be the change in the type of nanoparticle distribution, which is clear from Figure 2, where the nanoparticle distribution is found to be top-heavy when $J=+1$ and bottom-heavy for $J=-1$. A destabilizing behavior of $R n$ for topheavy nanoparticle distribution was reported earlier by Nield and Kuznetsov [2]. Moreover, for a bottom-heavy 
(a)-(1)

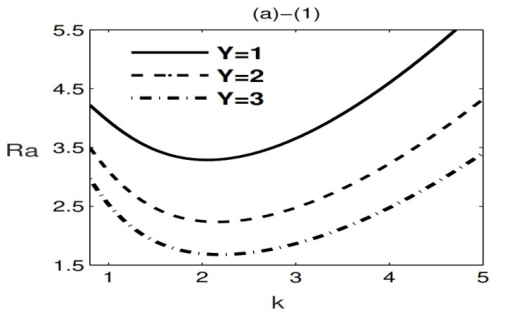

(a) $-(2)$

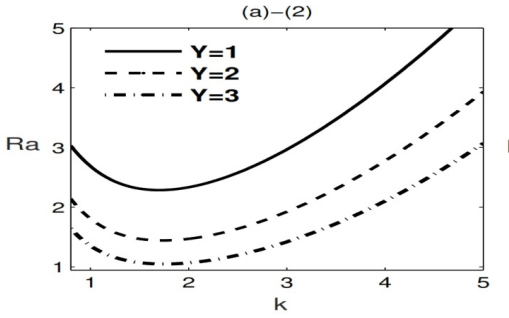

(a) $-(3)$

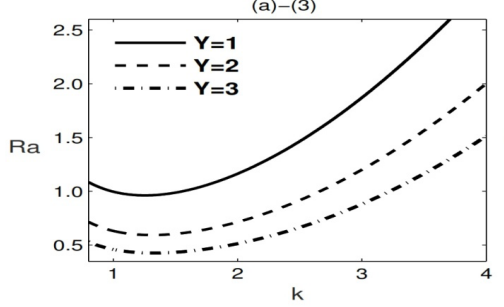

(b) -(1)

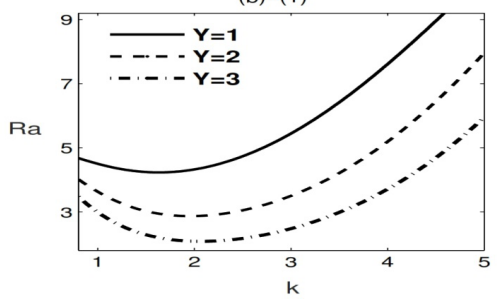

(b) $-(2)$

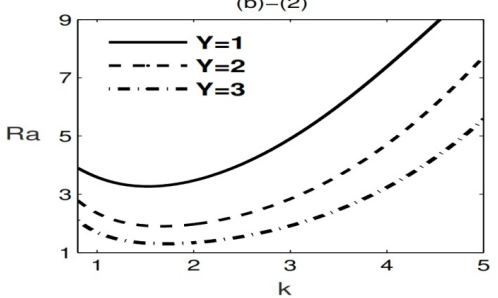

(b) $-(3)$

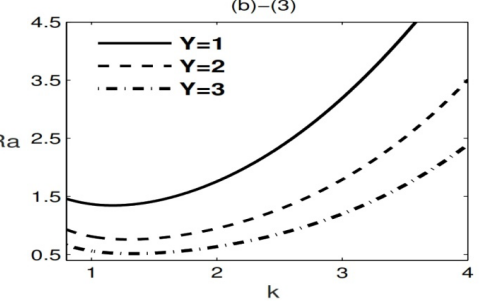

Figure 3: Neutral stability curves for distinct values of $Y$ for (a) $J=+1$ and (b) $J=-1$; for (1) I_C-I_C, (2) I_C-I_CHF, and (3) I_C-F_CHF boundaries.
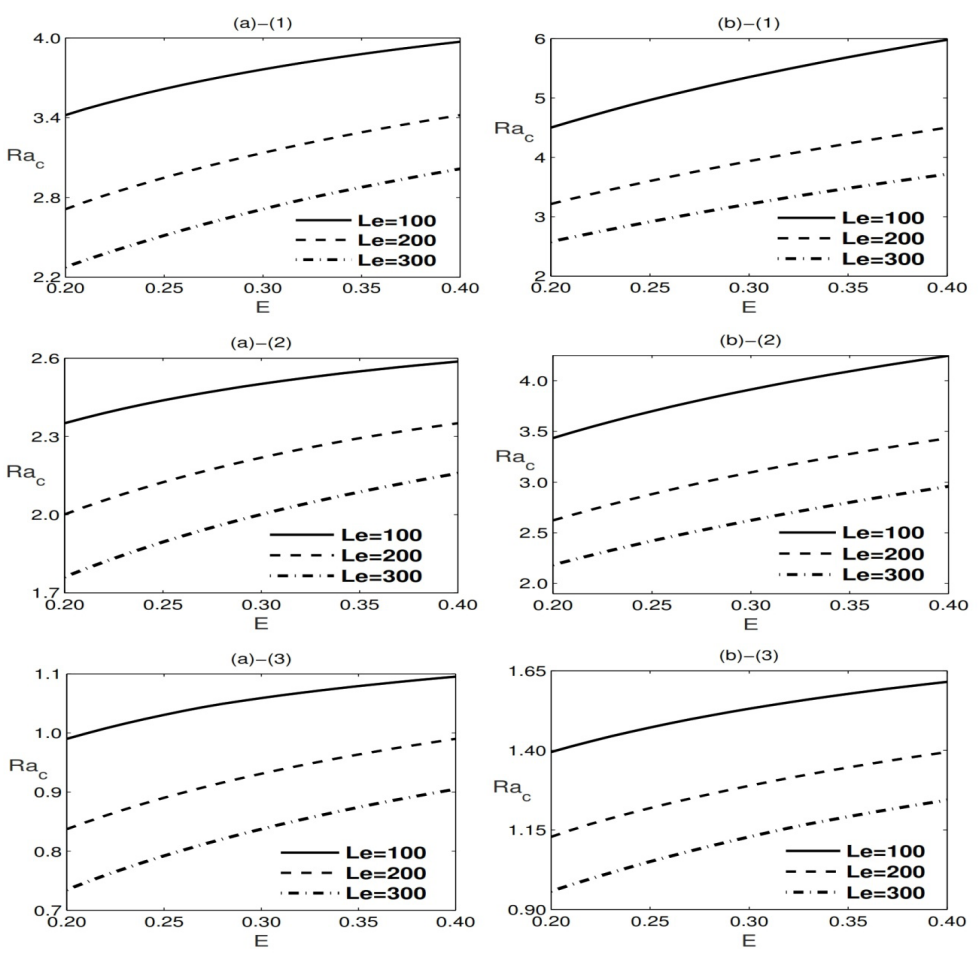

Figure 4: Variation of $R a$ as a function of $E$ for distinct values of $L e$ for (a) $J=+1$ and (b) $J=-1$; for (1) I_C-I_C, (2) I_C-I_CHF, and (3) I_C-F_CHF boundaries. 

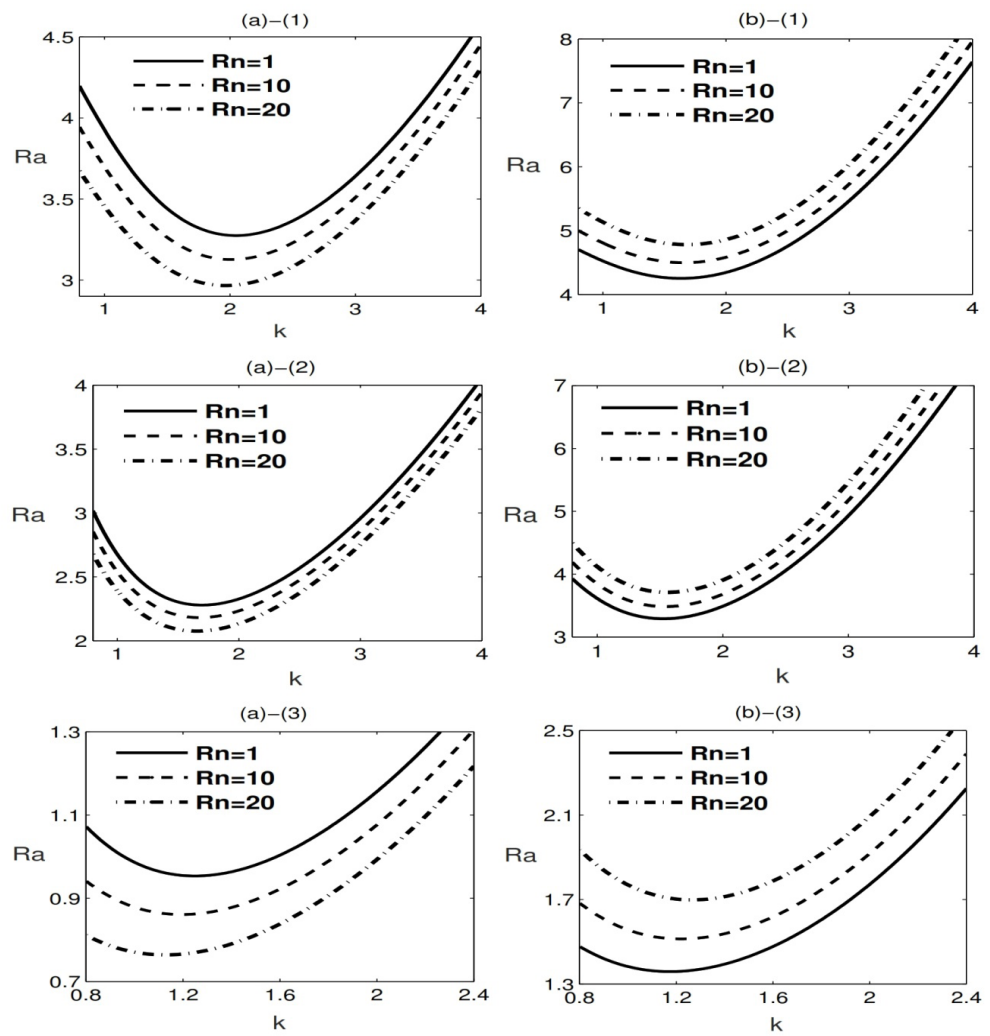

Figure 5: Neutral stability curves for distinct values of $R n$ for (a) $J=+1$ and (b) $J=-1$; for (1) I_C-I_C, (2) I_C-I_CHF, and (3) I_C-F_CHF boundaries.
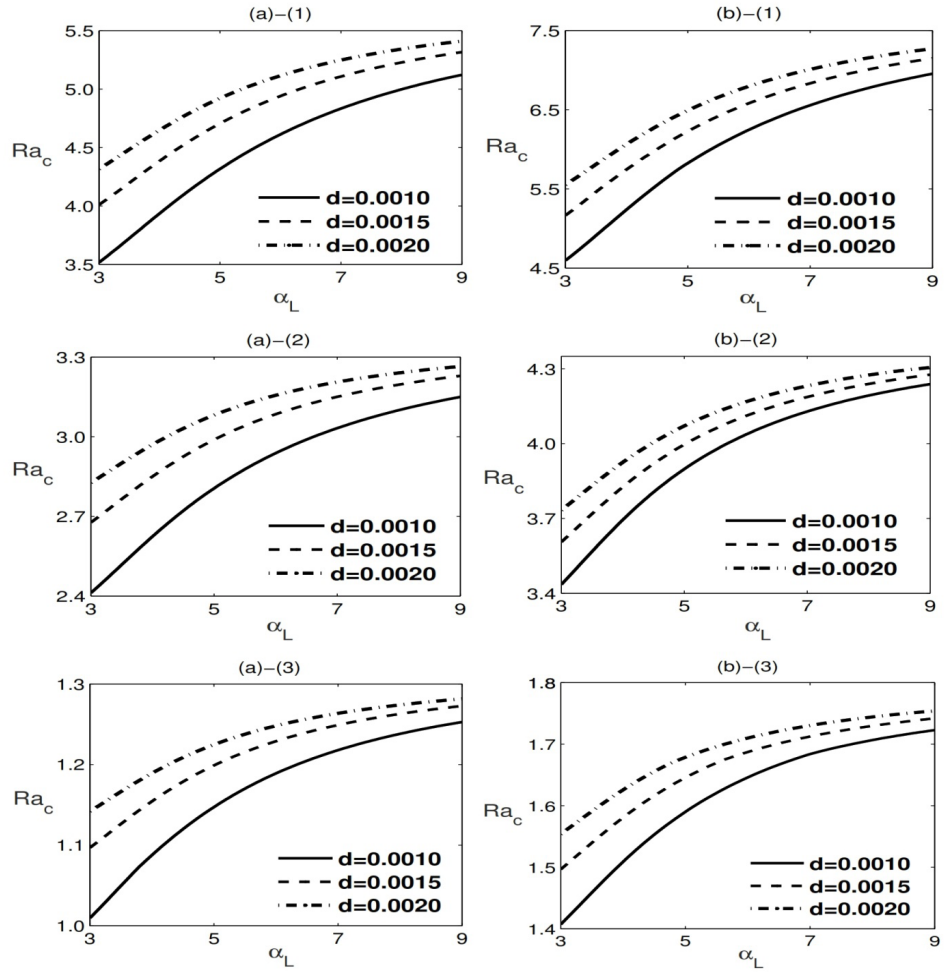

Figure 6: Variation of $R a_{c}$ as a function of $\alpha_{L}$ for distinct values of $d$ for (a) $J=+1$ and (b) $J=-1$; for (1) I_C-I_C, (2) I_C-I_CHF, and (3) I_C-F_CHF boundaries. 

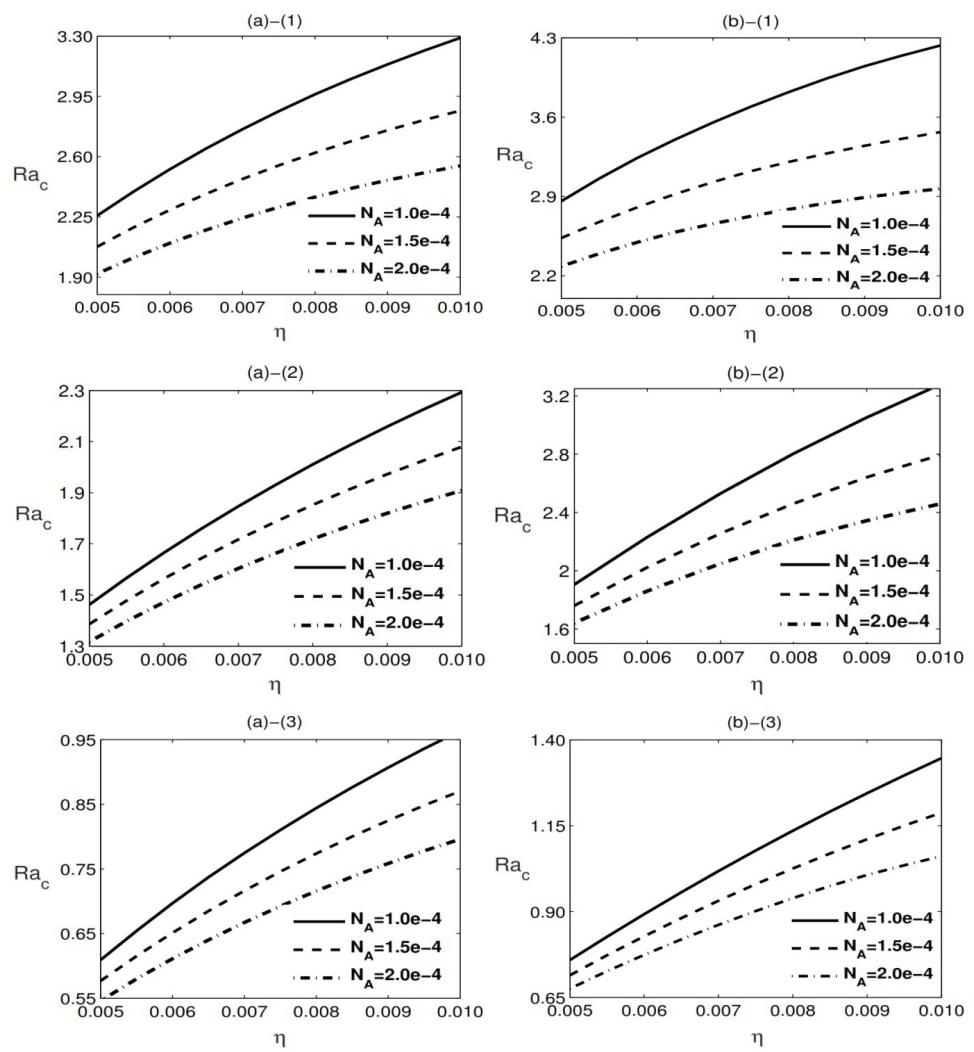

Figure 7: Variation of $R a_{c}$ as a function of $\eta$ for distinct values of $N_{A}$ for (a) $J=+1$ and (b) $J=-1$; for (1) I_C I_C, (2) I_C.I_CHF, and (3) I_C-F_CHF boundaries.

nanoparticle distribution, a stabilizing effect of $R n$ was observed in various studies including [16, 44].

In order to unfurl the key features of Langevin parameter $\alpha_{L}$ and the width of nanofluid layer $d$, the influence of both $\alpha_{L}$ and $d$ on $R a_{c}$ is presented in Figure 6. We note that the value of $R a_{c}$ increases when $\alpha_{L}$ increases. This is so because, in the process of MNF convection, the buoyancy forces together with the forces that originate because of the appearance of an internal heat source dominate the magnetic forces, and, hence as the intensity of magnetic field increases, the disturbance in the MNF layer decelerates. This leads to a higher value of $R a_{c}$. As plotted in Figure 6, the value of $R a_{c}$ is found to be increasing with an increase in the value of $d$. As the value of $d$ increase, the value of critical temperature $\Delta T_{c}$ decreases which in turn suppresses the disturbance in the magnetic nanofluid layer and tends to a higher value of $R a_{c}$. Thus both the parameters $\alpha_{L}$ and $d$ delay the onset of MNF convection. In the absence of magnetic nanoparticles, a similar behavior was observed for $\alpha_{L}$ and $d$ by Kaloni and Lou [39].

Figure 7 shows the effect of $\eta$ and $N_{A}$ on $R a_{c}$ for three different boundary conditions. It can be seen that
$R a_{c}$ decreases as the value of $N_{A}$ increases. On the other hand, $R a_{c}$ increases with an increase in $\eta$. As the value of $N_{A}$ increases, the value of thermophoretic diffusivity also increases. At a higher value of thermophoretic diffusivity, thermophoresis promotes the growth of turbulence in the MNF layer, which gives a lower value of $R a_{c}$. Thus the parameter $\eta$ has a stabilizing effect and $N_{A}^{c}$ has a destabilizing effect on the system. An identical behavior of the parameter $N_{A}$ was reported earlier by Yadav et al. [7] in the absence of magnetic field. For the parameter $\eta$, a similar behavior was reported earlier by Hill [13] for a regular fluid.

Table 2 presents the values of $k_{c}$ and $R a_{c}$ for three different boundaries, namely, I_C-I_C, I_C-I_CHF, and I_C-F_CHF. These values are calculated for two different configurations, that is, $J=+1$ and $J=-1$. Our main interest in presenting this table is to examine the influence of $Y$ and $\eta$ on the critical wave number $k_{c}$ and the critical thermal Rayleigh number $R a_{c}$. The table shows that the value of $R a_{c}$ increases as $\eta$ increases, whereas, $R a_{c}$ decreases as $Y$ increases. This behavior is noticed to be the same for both the two different configurations and all the three boundary conditions. Thus the parameter $Y$ 
Table 2: The values of the $k_{c}$ and $R a_{c}$ for three different boundary conditions.

\begin{tabular}{|c|c|c|c|c|c|c|c|c|}
\hline & & & I_C-I_C & & I_C-I & & I_C-F & \\
\hline$J$ & $Y$ & $\eta$ & $k_{c}$ & $R a_{c}$ & $k_{c}$ & $R a_{c}$ & $k_{c}$ & $R a_{c}$ \\
\hline \multirow[t]{12}{*}{+1} & 1 & 0.001 & 2.23 & 0.6144 & 1.76 & 0.3656 & 1.35 & 0.1476 \\
\hline & & 0.004 & 2.18 & 1.9434 & 1.74 & 1.2343 & 1.32 & 0.5100 \\
\hline & & 0.007 & 2.12 & 2.7561 & 1.72 & 1.8437 & 1.29 & 0.7712 \\
\hline & & 0.010 & 2.05 & 3.2883 & 1.70 & 2.2875 & 1.25 & 0.9622 \\
\hline & 5 & 0.001 & 2.22 & 0.1298 & 1.76 & 0.0761 & 1.36 & 0.0304 \\
\hline & & 0.004 & 2.23 & 0.4957 & 1.76 & 0.2934 & 1.35 & 0.1176 \\
\hline & & 0.007 & 2.22 & 0.8236 & 1.76 & 0.4938 & 1.35 & 0.1980 \\
\hline & & 0.001 & 2.22 & 1.1151 & 1.76 & 0.6776 & 1.34 & 0.2717 \\
\hline & 10 & 0.001 & 2.22 & 0.0653 & 1.76 & 0.0382 & 1.36 & 0.0153 \\
\hline & & 0.004 & 2.23 & 0.2549 & 1.76 & 0.1497 & 1.35 & 0.0597 \\
\hline & & 0.007 & 2.23 & 0.4337 & 1.76 & 0.2561 & 1.35 & 0.1019 \\
\hline & & 0.010 & 2.23 & 0.6013 & 1.76 & 0.3574 & 1.35 & 0.1418 \\
\hline \multirow[t]{12}{*}{-1} & 1 & 0.001 & 2.18 & 0.6640 & 1.75 & 0.3920 & 1.35 & 0.1554 \\
\hline & & 0.004 & 2.01 & 2.4104 & 1.69 & 1.5500 & 1.3 & 0.6131 \\
\hline & & 0.007 & 1.82 & 3.5526 & 1.61 & 2.5300 & 1.24 & 1.0167 \\
\hline & & 0.010 & 1.63 & 4.2320 & 1.53 & 3.2718 & 1.17 & 1.3456 \\
\hline & 5 & 0.001 & 2.22 & 0.1322 & 1.76 & 0.0773 & 1.36 & 0.0308 \\
\hline & & 0.004 & 2.20 & 0.5312 & 1.75 & 0.3117 & 1.35 & 0.1238 \\
\hline & & 0.007 & 2.17 & 0.9254 & 1.74 & 0.5477 & 1.35 & 0.2172 \\
\hline & & 0.010 & 2.15 & 1.3071 & 1.74 & 0.7831 & 1.35 & 0.3106 \\
\hline & 10 & 0.001 & 2.22 & 0.0659 & 1.76 & 0.0385 & 1.36 & 0.0154 \\
\hline & & 0.004 & 2.21 & 0.2647 & 1.76 & 0.1547 & 1.36 & 0.0615 \\
\hline & & 0.007 & 2.21 & 0.4636 & 1.75 & 0.2712 & 1.36 & 0.1076 \\
\hline & & 0.010 & 2.20 & 0.6614 & 1.75 & 0.3879 & 1.36 & 0.1537 \\
\hline
\end{tabular}

always hastens the onset of convection and the parameter $\eta$ always decelerates the onset of convection. These observations agree well with the findings of Hill [13] [see Table 2, pp. 462], where the author investigated this problem in the absence of nanoparticles and magnetic field. It is also noticed that the value of $R a_{c}$ is higher in case of I_C-I_C boundary condition and the least for I_C-F_ CHF boundary condition for both $J=+1$ and $J=-1$. Thus the system is most stable for I_C-I_C boundaries and least stable for I_C-F_CHF boundaries.

\section{Conclusions}

The linear stability theory is applied to study the magnetic nanofluid convection stimulated by selective absorption of radiation subjected to an applied magnetic field. The problem is investigated for two different configurations, namely, when the layer is heated from below $(J=+1)$ and when the layer is heated from above $(J=-1)$. The Chebyshev pseudospectral method is applied to solve the resulting eigenvalue problem for I_C-I_C, I_C-I_CHF, and I_C-F_CHF boundaries. The effect of the porosity parameter $E$, the parameter $Y$, the Lewis number $L e$, 
the concentration Rayleigh number $R n$, the Langevin parameter $\alpha_{L}$, the width of nanofluid layer $d$, the diffusivity ratio $\eta$, and the modified diffusivity ratio $N_{A}$ is observed at the onset of convection. The following conclusions are drawn:

(1) The value of $R a_{c}$ increases as the value of $E, \alpha_{L}, d$, and $\eta$ increases, and decreases as the value of $Y, L e$, and $N_{A}$ increases. Thus, $E, \alpha_{L}, d$, and $\eta$ decelerate the onset of MNF convection, and $Y, L e$, and $N_{A}$ promote the onset of MNF convection.

(2) The effect of $R n$ on the onset of convection depends on the type of configuration (i.e., whether $J=+1$ or $J=-1$ ). For $J=+1, R n$ hasten the onset of MNF convection, whereas for $J=-1, R n$ delays the onset of MNF convection.

(3) Among all the three type of boundaries, the system is found to be most stable for I_C-I_C boundary condition and least stable for I_C-F_CHF boundary condition.

Acknowledgment: The work in this article is supported by Council of Scientific and Industrial Research (CSIR), New Delhi, in the form of Research and Development project [Ref. No. 25(0255)/16/EMR-II]. The authors gratefully acknowledge the support thus received.

\section{References}

[1] Buongiorno, J. (2006). Convective transport in nanofluids. Journal of heat transfer, 128(3), 240-250.

[2] Nield, D.A., Kuznetsov, A.V. (2009). Thermal instability in a porous medium layer saturated by a nanofluid. International Journal of Heat and Mass Transfer, 52(25-26), 5796-5801.

[3] Nield, D.A., Kuznetsov, A.V. (2014). Thermal instability in a porous medium layer saturated by a nanofluid: a revised model. International Journal of Heat and Mass Transfer, 68, 211-214.

[4] Yadav, D., Agarwal, G., Lee, J. (2016). Thermal instability in a rotating nanofluid layer: a revised model. Ain Shams Engineering Journal, 7(1), 431-440.

[5] Bhadauria, B.S., Agarwal, S. (2011). Convective transport in a nanofluid saturated porous layer with thermal non equilibrium model. Transport in porous media, 88(1), 107-131.

[6] Yadav, D., Bhargava, R., Agarwal, G. S. (2012). Boundary and internal heat source effects on the onset of DarcyBrinkman convection in a porous layer saturated by nanofluid. International Journal of Thermal Sciences, 60, 244-254.

[7] Yadav, D., Lee, J., Cho, H.H. (2015). Brinkman convection induced by purely internal heating in a rotating porous medium layer saturated by a nanofluid. Powder Technology, 286, 592-601.

[8] Yadav, D., Wang, J. (2018). Convective Heat Transport in a Heat Generating Porous Layer Saturated by a Non-Newtonian Nanofluid. Heat Transfer Engineering, 1-20.
[9] Yadav, D. (2018). The Influence of Pulsating Throughflow on the Onset of Electro-Thermo-Convection in a Horizontal Porous Medium Saturated by a Di-electric Nanofluid. Journal of Applied Fluid Mechanics, 11(6), 1679-1689.

[10] Krishnamurti, R. (1998). Convection induced by selective absorption of radiation: a laboratory model of conditional instability. Dynamics of atmospheres and oceans, 27(1-4), 367382.

[11] Hill, A.A. (2004). Penetrative convection induced by the absorption of radiation with a nonlinear internal heat source. Dynamics of atmospheres and oceans, 38(1), 57-67.

[12] Straughan, B. (2002). Global stability for convection induced by absorption of radiation. Dynamics of atmospheres and oceans, 35(4), 351-361.

[13] Hill, A.A. (2003). Convection due to the selective absorption of radiation in a porous medium. Continuum Mechanics and Thermodynamics, 15(5), 451-462.

[14] Hill, A.A. (2004). Convection induced by the selective absorption of radiation for the brinkman model. Continuum Mechanics and Thermodynamics, 16(1-2), 43-52.

[15] Chang, M.H. (2004). Stability of convection induced by selective absorption of radiation in a fluid overlying a porous layer. Physics of Fluids, 16(10), 3690-3698.

[16] Gupta, U., Ahuja, J., Wanchoo, R. (2013). Magneto convection in a nanofluid layer. International Journal of Heat and Mass Transfer, 64, 1163-1171.

[17] Yadav, D., Bhargava, R., Agarwal, G. S. (2013). Thermal instability in a nanofluid layer with a vertical magnetic field. Journal of Engineering Mathematics, 80(1), 147-164.

[18] Murthy, P. V. S. N., RamReddy, C., Chamkha, A. J., Rashad, A. M. (2013). Magnetic effect on thermally stratified nanofluid saturated non-Darcy porous medium under convective boundary condition. International Communications in Heat and Mass Transfer, 47, 41-48.

[19] Yadav, D., Bhargava, R., Agrawal, G.S., Hwang, G.S., Lee, J., Kim, M.C. (2014). Magneto-convection in a rotating layer of nanofluid. Asia-Pacific Journal of Chemical Engineering, 9(5), 663-677.

[20] Yadav, D., Kim, C., Lee, J., Cho, H.H. (2015). Influence of magnetic field on the onset of nanofluid convection induced by purely internal heating. Computers \& Fluids, 121, 26-36.

[21] Yadav, D., Wang, J., Bhargava, R., Lee, J., Cho, H.H. (2016). Numerical investigation of the effect of magnetic field on the onset of nanofluid convection. Applied Thermal Engineering, 103, 1441-1449.

[22] Sheikholeslami, M. (2018). CuO-water nanofluid flow due to magnetic field inside a porous media considering Brownian motion. Journal of Molecular Liquids, 249, 921-929.

[23] Borglin, S.E., Moridis, G.J., Oldenburg, C.M. (2000). Experimental studies of the flow of ferrofluid in porous media. Transport in Porous Media, 41(1), 61-80.

[24] Vaidyanathan, G., Sekar, R., Balasubramanian, R. (1991). Ferroconvective instability of fluids saturating a porous medium. International journal of engineering science, 29(10), 1259-1267.

[25] Mahajan, A., Sharma, M.K. (2014). Convection in magnetic nanofluids in porous media. Journal of Porous Media, 17(5), 439455. 
[26] Sheikholeslami, M. (2017). Numerical simulation of magnetic nanofluid natural convection in porous media. Physics Letters A, 381(5), 494-503.

[27] Sharma, M.K., Singh, R. (2014). Linear stability analysis of double-diffusive convection in magnetic nanofluids in porous media. Journal of Porous Media, 17(10), 883-900.

[28] Sheikholeslami, M., Rashidi, M.M., Hayat, T., Ganji, D.D. (2016). Free convection of magnetic nanofluid considering MFD viscosity effect. Journal of Molecular Liquids, 218, 393-399.

[29] Sheikholeslami, M. (2016). CVFEM for magnetic nanofluid convective heat transfer in a porous curved enclosure. The European Physical Journal Plus, 131(11), 413.

[30] Mahajan, A., Sharma, M.K. (2017). Penetrative convection in magnetic nanofluids via internal heating. Physics of Fluids, 29(3), 034101.

[31] Mahajan, A., Sharma, M.K. (2018). The onset of penetrative convection stimulated by internal heating in a magnetic nanofluid saturating a rotating porous medium. Canadian Journal of Physics, 96(8), 898-911.

[32] Sheikholeslami, M., Rokni, H. B. (2018). Magnetic nanofluid flow and convective heat transfer in a porous cavity considering Brownian motion effects. Physics of Fluids, 30(1), 012003.

[33] Mahajan, A., Sharma, M.K. (2018). Penetrative convection in an internally heated layer of magnetic nanofluid saturating a porous medium. Magnetohydrodynamics, 54(3), 225-243.

[34] Vasseur, P., Satish, M.G., Robillard, L. (1987). Natural convection in a thin, inclined, porous layer exposed to a constant heat flux. International Journal of Heat and Mass Transfer, 30(3), 537-549.

[35] Reda, D.C. (1983). Natural convection experiments in a liquidsaturated porous medium bounded by vertical coaxial cylinders. Journal of heat transfer, 105(4), 795-802.

[36] Prasad, V., Kulacki, F.A., Kulkarni, A.V. (1986). Free convection in a vertical, porous annulus with constant heat flux on the inner wall-experimental results. International journal of heat and mass transfer, 29(5), 713-723.

[37] Shliomis, M.I., Smorodin, B.L. (2002). Convective instability of magnetized ferrofluids. Journal of Magnetism and Magnetic Materials, 252, 197-202.

[38] Mahajan, A., Sharma, M.K. (2018). Convection in a magnetic nanofluid saturating a porous medium under the influence of a variable gravity field. Engineering Science and Technology, an International Journal, 21(3), 439-450.

[39] Kaloni, P.N., Lou, J.X. (2004). Convective instability of magnetic fluids. Physical Review E, 70(2), 026313.

[40] Finlayson, B.A. (1970). Convective instability of ferromagnetic fluids. Journal of Fluid Mechanics, 40(4), 753-767.

[41] Rosensweig, R.E. (1997). Ferrohydrodynamics. Dover Publications, INC. Mineola, New York.

[42] Yadav, D., Lee, D., Cho, H.H., Lee, J. (2016). The onset of doublediffusive nanofluid convection in a rotating porous medium layer with thermal conductivity and viscosity variation: a revised model. Journal of Porous media, 19(1), 31-46.

[43] Awasthi, M.K., Kumar, V., Patel, R.K., (2016). Onset of triply diffusive convection in a maxwell fluid saturated porous layer with internal heat source. Ain Shams Engineering Journal, 9(4), 1591-1600.

[44] Gupta, U., Sharma, J., Sharma, V. (2015). Instability of binary nanofluids with magnetic field. Applied Mathematics and Mechanics, 36(6), 693-706. 